\title{
Evaluation of teriparatide treatment in adults with osteogenesis imperfecta
}

\author{
Eric S. Orwoll, ${ }^{1}$ Jay Shapiro, ${ }^{2}$ Sandra Veith, ${ }^{1}$ Ying Wang, ${ }^{1}$ Jodi Lapidus, ${ }^{1}$ \\ Chaim Vanek, ${ }^{1}$ Jan L. Reeder, ${ }^{1}$ Tony M. Keaveny, ${ }^{3,4}$ David C. Lee, ${ }^{3}$ Mary A. Mullins, ${ }^{5}$ \\ Sandesh C.S. Nagamani, 5 and Brendan Lee ${ }^{5,6}$ \\ 10regon Health and Science University, Portland, Oregon, USA. ${ }^{2}$ Kennedy Krieger Institute, Baltimore, Maryland, USA. \\ ${ }^{3}$ ON Diagnostics, Berkeley, California, USA. ${ }^{4}$ Departments of Mechanical Engineering and Bioengineering, UC Berkeley, Berkeley, California, USA. \\ ${ }^{5}$ Baylor College of Medicine, Houston, Texas, USA. ${ }^{6}$ Howard Hughes Medical Institute, Houston, Texas, USA.
}

\begin{abstract}
Background. Adults with osteogenesis imperfecta (OI) have a high risk of fracture. Currently, few treatment options are available, and bone anabolic therapies have not been tested in clinical trials for OI treatment.

Methods. 79 adults with OI were randomized to receive $20 \mu$ g recombinant human parathyroid hormone (teriparatide) or placebo for 18 months in a double-blind, placebo-controlled trial. The primary endpoint was the percent change in areal bone mineral density (aBMD) of the lumbar spine (LS), as determined by dualenergy $\mathrm{X}$-ray absorptiometry. Secondary endpoints included percent change in bone remodeling markers and vertebral volumetric $B M D$ (vBMD) by quantitative computed tomography, estimated vertebral strength by finite element analysis, and self-reported fractures.
\end{abstract}

Results. Compared with the placebo group, the teriparatide group showed increased LS aBMD $(6.1 \% \pm 1.0 \%$ vs. $2.8 \% \pm 1.0 \%$ change from baseline; $P<0.05)$ and total hip aBMD $(2.6 \% \pm 1.0 \%$ vs. $-2.4 \% \pm 1.0 \%$ change; $P<0.001)$. Vertebral vBMD and strength improved with teriparatide therapy $(18 \% \pm 6 \%$ and $15 \% \pm 3 \%$ change, respectively), but declined with placebo $(-5.0 \% \pm 6 \%$ and $-2.0 \% \pm 3 \%$ change; $P<0.05$ for both comparisons). Serum procollagen type $1 \mathrm{~N}$-terminal propeptide (P1NP) and urine collagen $\mathrm{N}$-telopeptide (NTx) levels increased with teriparatide therapy $(135 \% \pm 14 \%$ and $64 \% \pm 10 \%$ change, respectively). Teriparatide-induced elevation of P1NP levels was less pronounced in severe forms of OI (type III/IV) compared with the milder form (type I). Type I OI patients exhibited robust BMD increases with teriparatide; however, there was no observed benefit for those with type III/IV OI. There was no difference in self-reported fractures between the 2 groups.

Conclusions. Adults with OI, particularly those with less severe disease (type I), displayed a teriparatide-induced anabolic response, as well as increased hip and spine aBMD, vertebral vBMD, and estimated vertebral strength.

Trial registration. Clinicaltrials.gov NCT00131469.

Funding. The Osteoporosis Imperfecta Foundation, Eli Lilly and Co., the National Center for Advancing Translational Science (NCATS) at the NIH (grant no. UL1RR024140), and the Baylor College of Medicine General Clinical Research Center (grant no. RR00188).

\section{Introduction}

Osteogenesis imperfecta (OI), the most common skeletal dysplasia, is caused by mutations in the genes encoding type 1 collagen (COL1A1 and COL1A2), its modifying enzymes, chaperone proteins, or signaling proteins (1). Although mutations in many genes have been found to cause OI, $85 \%-90 \%$ of patients have mutations in type 1 collagen (2). The mutations alter the properties of bone matrix and affect osteoblast function and bone remodeling, leading to impaired bone quality, mass, and structure $(3,4)$. Fractures

Conflict of interest: Eric S. Orwoll consults with Eli Lilly and Co. Tony M. Keaveny consults for and has personal financial equity with ON Diagnostics. David C. Lee is a full-time employee of ON Diagnostics.

Role of funding source: The study was designed, conducted, analyzed, and reported entirely by the authors. The Osteogenesis Imperfecta Foundation provided funding support through its Seed Grant for Clinical Research program. Eli Lilly and Co. provided funding and study drug through its investigator-initiated support program. Resources for study conduct were provided by the Clinical and Translational Research Center of the Oregon Clinical and Translational Research Institute and the Baylor College of Medicine General Clinical Research Center.

Citation for this article: J Clin Invest. 2014;124(2):491-498. doi:10.1172/JCI71101. and skeletal deformity commonly occur in childhood, but fracture risk remains high in adulthood.

In children, treatment with bisphosphonates is associated with reduced bone turnover, higher bone mineral density (BMD), improved structure, and, in some studies, lower fracture rate (5-13). In adults, there have been few treatment evaluations, but observational studies and 2 small randomized trials suggest that although bisphosphonates lead to increased $\operatorname{BMD}(6,14,15)$, a significant reduction in fracture rate has not been demonstrated with either oral or intravenous administration. Bone strength could also be increased by anabolic therapy that improves bone formation, mass, and structure; however, such therapy has not been evaluated in OI.

Teriparatide (human parathyroid hormone [PTH]) is a bone anabolic therapy for osteoporosis that increases bone remodeling, formation, and density; improves bone microstructure, including increased trabecular number and thickness; and reduces fracture risk (16-18). Here we report the results of a prospective, randomized, proof-of-concept trial designed to assess the ability of 
Table 1

Participant demographic and baseline characteristics

\begin{tabular}{|c|c|c|c|c|}
\hline Characteristic & Total $(n=78)$ & Treatment $(n=38)$ & Placebo $(n=40)$ & $P$ \\
\hline $\begin{array}{l}\text { Age (yr), mean } \pm S D \\
\text { Range }\end{array}$ & $\begin{array}{c}41.0 \pm 11.5 \\
18-75\end{array}$ & $\begin{array}{c}40.8 \pm 12.9 \\
18-66\end{array}$ & $\begin{array}{l}41.2 \pm 10.1 \\
23-75\end{array}$ & 0.88 \\
\hline Male, $n(\%)$ & $32(41.6 \%)$ & $16(42.1 \%)$ & $16(41.0 \%)$ & 0.92 \\
\hline Height $(m)$, mean \pm SD & $1.5 \pm 0.3$ & $1.5 \pm 0.3$ & $1.5 \pm 0.3$ & 0.80 \\
\hline $\mathrm{BMI}\left(\mathrm{kg} / \mathrm{m}^{2}\right)$, mean $\pm \mathrm{SD}$ & $31.2 \pm 16.9$ & $31.1 \pm 19.8$ & $31.4 \pm 13.7$ & 0.95 \\
\hline $\begin{array}{l}\text { OI type }, n(\%) \\
\text { I } \\
\text { III } \\
\text { IV }\end{array}$ & $\begin{array}{l}51(66.2 \%) \\
14(18.2 \%) \\
12(15.6 \%)\end{array}$ & $\begin{array}{l}24(63.2 \%) \\
7(18.4 \%) \\
7(18.4 \%)\end{array}$ & $\begin{array}{l}27(69.2 \%) \\
7(18.0 \%) \\
5(12.8 \%)\end{array}$ & 0.78 \\
\hline $\begin{array}{l}\text { Number of lifetime fractures, } n(\%) \\
\quad 0-15 \\
16-35 \\
36-60 \\
>61\end{array}$ & $\begin{array}{l}20(26.0 \%) \\
27(35.1 \%) \\
17(22.1 \%) \\
13(16.9 \%)\end{array}$ & $\begin{array}{l}13(34.2 \%) \\
12(31.6 \%) \\
6(15.8 \%) \\
7(18.4 \%)\end{array}$ & $\begin{array}{c}7(18.0 \%) \\
15(38.5 \%) \\
11(28.2 \%) \\
6(15.4 \%)\end{array}$ & 0.30 \\
\hline $\begin{array}{l}\text { 24-hour urine calcium }(\mathrm{mg} / 24 \mathrm{~h}) \text {, mean } \pm \mathrm{SD} \\
\end{array}$ & $\begin{array}{c}154.5 \pm 88.7 \\
73\end{array}$ & $\begin{array}{c}139.0 \pm 78.1 \\
36\end{array}$ & $\begin{array}{c}169.6 \pm 96.6 \\
37\end{array}$ & 0.14 \\
\hline $\begin{array}{l}\text { 25-hydroxy }(\mathrm{OH}) \text { vitamin } \mathrm{D}(\mathrm{ng} / \mathrm{ml}) \text {, mean } \pm \mathrm{SD} \\
\end{array}$ & $\begin{array}{l}33.2 \pm 13.4 \\
76\end{array}$ & $\begin{array}{c}32.7 \pm 11.5 \\
38\end{array}$ & $\begin{array}{c}33.8 \pm 15.2 \\
38\end{array}$ & 0.72 \\
\hline$\underset{n}{\operatorname{Serum}} \mathrm{PTH}(\mathrm{pg} / \mathrm{ml})$, mean \pm SD & $\begin{array}{l}29.3 \pm 14.8 \\
75\end{array}$ & $\begin{array}{l}30.2 \pm 16.3 \\
37\end{array}$ & $\begin{array}{l}28.4 \pm 13.4 \\
38\end{array}$ & 0.61 \\
\hline$\underset{n}{\text { Serum phosphorus }(p g / m l), \text { mean } \pm S D}$ & $\begin{array}{l}3.6 \pm 0.6 \\
76\end{array}$ & $\begin{array}{l}3.5 \pm 0.5 \\
38\end{array}$ & $\begin{array}{l}3.7 \pm 0.7 \\
38\end{array}$ & 0.07 \\
\hline $\begin{array}{l}\text { Creatinine }(\mathrm{mg} / \mathrm{dl}), \text { mean } \pm \mathrm{SD} \\
\end{array}$ & $\begin{array}{l}0.7 \pm 0.3 \\
76\end{array}$ & $\begin{array}{l}0.7 \pm 0.2 \\
38\end{array}$ & $\begin{array}{l}0.7 \pm 0.3 \\
38\end{array}$ & 0.96 \\
\hline$\underset{n}{\text { Calcium }(\mathrm{mg} / \mathrm{dl}) \text {, mean } \pm \mathrm{SD}}$ & $\begin{array}{l}9.5 \pm 0.4 \\
76\end{array}$ & $\begin{array}{l}9.4 \pm 0.3 \\
38\end{array}$ & $\begin{array}{l}9.6 \pm 0.5 \\
38\end{array}$ & 0.20 \\
\hline $\begin{array}{l}\text { P1NP }(\mu \mathrm{g} / \mathrm{l}), \text { mean } \pm \mathrm{SD} \\
\text { Range } \\
n\end{array}$ & $\begin{array}{c}34.7 \pm 25.0 \\
8.9-141.7 \\
71\end{array}$ & $\begin{array}{c}37.7 \pm 28.8 \\
11.3-141.7 \\
\quad 35\end{array}$ & $\begin{array}{l}31.7 \pm 20.6 \\
8.9-125.5 \\
36\end{array}$ & 0.32 \\
\hline $\begin{array}{l}\text { Urine NTx (nM BCE/mM), mean } \pm \text { SD } \\
\text { Range } \\
n\end{array}$ & $\begin{array}{c}50.6 \pm 38.5 \\
10.6-191.9 \\
71\end{array}$ & $\begin{array}{c}50.8 \pm 29.6 \\
10.6-144.3 \\
35\end{array}$ & $\begin{array}{l}50.5 \pm 45.9 \\
13.2-191.9 \\
\quad 36\end{array}$ & 0.97 \\
\hline $\begin{array}{l}\text { FN BMD }\left(\mathrm{g} / \mathrm{cm}^{2}\right) \text {, mean } \pm \mathrm{SD} \\
n\end{array}$ & $\begin{array}{c}0.68 \pm 0.12 \\
58\end{array}$ & $\begin{array}{l}0.68 \pm 0.13 \\
27\end{array}$ & $\begin{array}{c}0.67 \pm 0.12 \\
31\end{array}$ & 0.79 \\
\hline $\begin{array}{l}\text { FN T score, mean } \pm \text { SD } \\
n\end{array}$ & $\begin{array}{c}-1.49 \pm 1.04 \\
58\end{array}$ & $\begin{array}{c}-1.45 \pm 1.09 \\
27\end{array}$ & $\begin{array}{c}-1.53 \pm 1.01 \\
31\end{array}$ & 0.79 \\
\hline 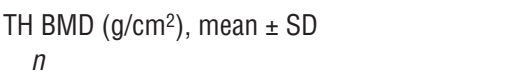 & $\begin{array}{l}0.79 \pm 0.15 \\
58\end{array}$ & $\begin{array}{l}0.82 \pm 0.16 \\
27\end{array}$ & $\begin{array}{l}0.76 \pm 0.14 \\
31\end{array}$ & 0.16 \\
\hline $\begin{array}{l}\text { TH T score, mean } \pm \mathrm{SD} \\
n\end{array}$ & $\begin{array}{l}-1.26 \pm 1.25 \\
58\end{array}$ & $\begin{array}{c}-1.01 \pm 1.32 \\
27\end{array}$ & $\begin{array}{c}-1.48 \pm 1.17 \\
31\end{array}$ & 0.16 \\
\hline 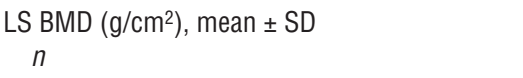 & $\begin{array}{l}0.75 \pm 0.12 \\
70\end{array}$ & $\begin{array}{l}0.77 \pm 0.10 \\
35\end{array}$ & $\begin{array}{c}0.74 \pm 0.13 \\
35\end{array}$ & 0.29 \\
\hline$\underset{n}{\mathrm{LS} T \text { score, mean } \pm \mathrm{SD}}$ & $\begin{array}{l}-2.68 \pm 1.06 \\
70\end{array}$ & $\begin{array}{c}-2.55 \pm 0.92 \\
35\end{array}$ & $\begin{array}{l}-2.82 \pm 1.18 \\
35\end{array}$ & 0.29 \\
\hline $\begin{array}{l}\text { Radial BMD }\left(\mathrm{g} / \mathrm{cm}^{2}\right) \text {, mean } \pm \mathrm{SD} \\
\end{array}$ & $\begin{array}{c}0.55 \pm 0.06 \\
51\end{array}$ & $\begin{array}{l}0.56 \pm 0.07 \\
\quad 28\end{array}$ & $\begin{array}{l}0.53 \pm 0.05 \\
23\end{array}$ & 0.14 \\
\hline $\begin{array}{l}\text { Vertebral vBMD }\left(\mathrm{mg} / \mathrm{cm}^{3}\right) \text {, mean } \pm \text { SD } \\
\text { S }\end{array}$ & $\begin{array}{c}1,262.7 \pm 613.5 \\
31\end{array}$ & $\begin{array}{c}1,167.0 \pm 573.0 \\
15\end{array}$ & $\begin{array}{c}1,352.4 \pm 654.8 \\
16\end{array}$ & 0.41 \\
\hline $\begin{array}{l}\text { Vertebral strength }(\mathrm{N}) \text {, mean } \pm \text { SD } \\
n\end{array}$ & $\begin{array}{l}3,562.9 \pm 1,101.5 \\
31\end{array}$ & $\begin{array}{c}3,472.3 \pm 983.1 \\
15\end{array}$ & $\begin{array}{c}3,647.8 \pm 1,228.2 \\
16\end{array}$ & 0.67 \\
\hline $\begin{array}{l}\Phi \text { (load/strength ratio), mean } \pm \text { SD } \\
n\end{array}$ & $\begin{array}{c}0.41 \pm 0.17 \\
31\end{array}$ & $\begin{array}{c}0.41 \pm 0.16 \\
15\end{array}$ & $\begin{array}{c}0.40 \pm 0.17 \\
16\end{array}$ & 0.85 \\
\hline
\end{tabular}

Aln 1 subject, OI type was unclassified. 


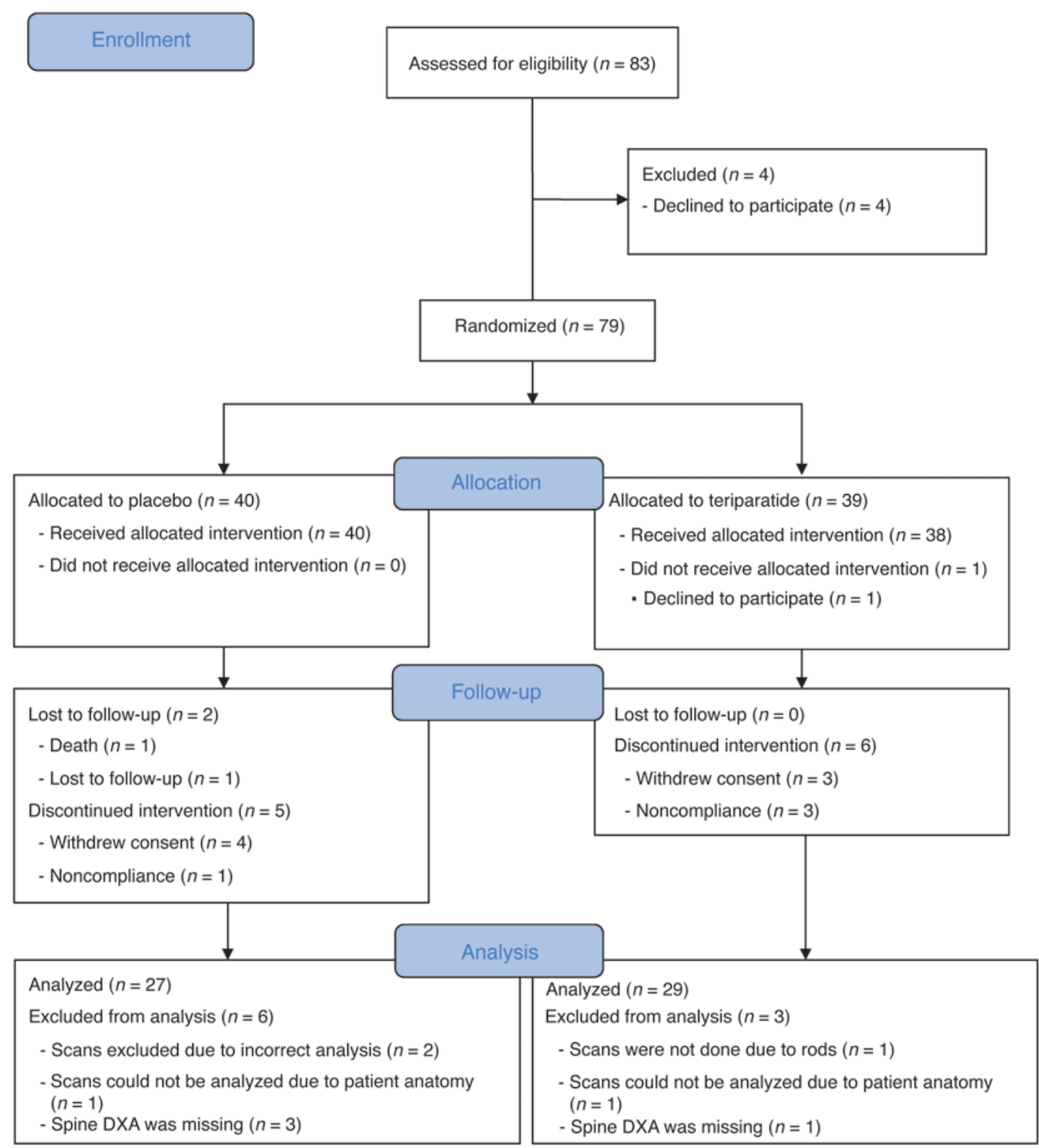

Figure 1

Enrollment, randomization, and study populations.

teriparatide to increase bone formation and positively affect skeletal mass and strength in adults with OI.

\section{Results}

79 patients were randomized. 1 patient discontinued participation before intervention and was not included in any analysis. The baseline characteristics of the 2 groups were similar (Table 1). 51 patients had type I OI, 14 had type III, and 12 had type IV; in 1 patient, OI could not be categorized. Mean T scores for areal BMD (aBMD) at the lumbar spine (LS), total hip (TH), and femoral neck (FN) were modestly reduced (Table 1 ). The number of patients with previous bisphosphonate use, the duration of use, and the distribution of OI subtypes treated were equal between groups. 7 patients in the placebo group and 6 in the teriparatide group discontinued the study, most commonly because of perceived inconvenience of the protocol (Figure 1). The modi- fied intent-to-treat (mITT) population included 32 teriparatidetreated and 33 placebo-treated participants. 1 death in the placebo group was considered to be unrelated to the trial.

Bone density and vertebral strength. In pooled analyses of all patients, treatment with teriparatide increased aBMD at the $\mathrm{TH}$, FN, and LS (Figure 2, A-C). At 18 months, the change in aBMD in the teriparatide group was higher than in the placebo group by $5.0 \%(\mathrm{TH}), 3.7 \%(\mathrm{FN})$, and $3.3 \%(\mathrm{LS})$ relative to baseline. Teriparatide yielded little change in radial BMD (Figure 2D). Treatmentinduced changes in bone mineral content (BMC) were essentially the same as those in $\mathrm{BMD}$, and there were no changes in bone area (data not shown). Vertebral vBMD declined in the placebo-treated group, but increased considerably in teriparatide-treated patients $(-4.7 \% \pm 5.7 \%$ vs. $18.3 \% \pm 5.9 \%$ change; $P<0.05$; Figure 3$)$. Similarly, the teriparatide group showed an increase in estimated vertebral strength compared with placebo, as assessed by finite element anal- 

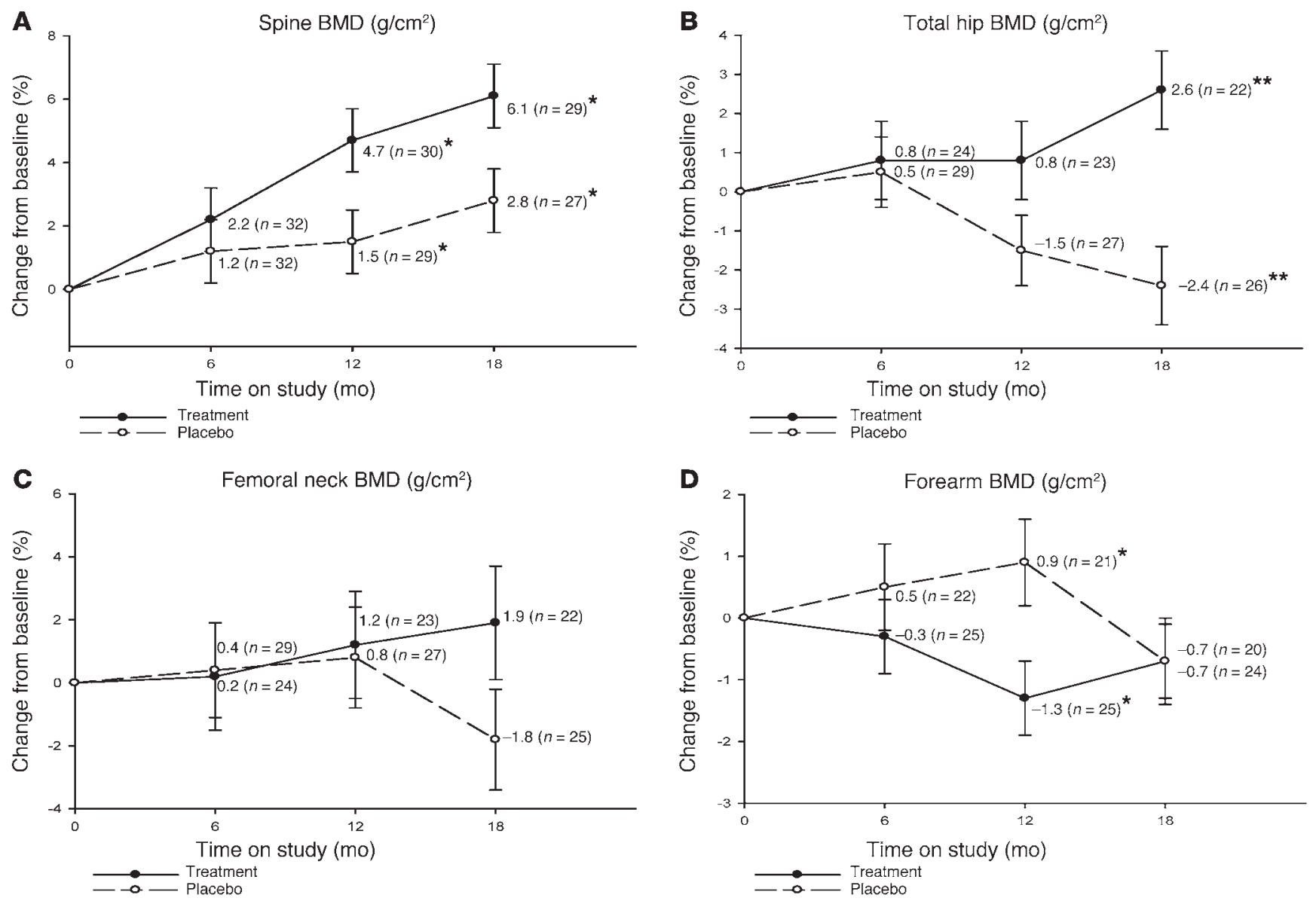

Figure 2

Bone density and vertebral strength. (A-D) Percent change from baseline in LS aBMD $\left(\mathrm{g} / \mathrm{cm}^{2} ; \mathbf{A}\right)$, TH aBMD $\left(\mathrm{g} / \mathrm{cm}^{2} ; \mathbf{B}\right), \mathrm{FN}$ aBMD $\left(\mathrm{g} / \mathrm{cm}^{2} ; \mathbf{C}\right)$, and radial aBMD $\left(\mathrm{g} / \mathrm{cm}^{2} ; \mathbf{D}\right)$ in teriparatide- and placebo-treated patients (mITT population). Error bars denote SEM. Values shown are estimated least-squares mean of percent change. The number of patients with nonmissing percent change data at each time point is shown in parentheses. ${ }^{\star} P<0.05,{ }^{* *} P<0.001$ between treatment groups.

ysis (FEA) $(15.2 \% \pm 3.5 \%$ vs. $-2.0 \% \pm 3.4 \%$ change; $P<0.05)$, whereas $\Phi($ load/strength ratio) declined $(-10.8 \% \pm 2.8 \%$ vs. $1.9 \% \pm 2.7 \%$ change; $P<0.05)$.

Post-hoc evaluation of OI subgroups revealed responses to therapy that were not apparent in the pooled patient analyses. In patients with type I OI, LS aBMD, the primary endpoint, changed marginally with placebo but increased with teriparatide treatment; LS aBMD was $5.2 \%$ higher in the teriparatide group at 18 months $(P=0.002$; Supplemental Figure 1A; supplemental material available online with this article; doi:10.1172/JCI71101DS1). There were similar effects on aBMD at the TH and FN (Supplemental Figure 1, B and C). Radial aBMD changed little in either group (Supplemental Figure 1D). In contrast, in patients with more severe OI (type III/VI), changes in aBMD were not significantly different in the treatment and placebo groups at any measurement site (Supplemental Figure 1, E-H). A test of a 3-way interaction (treatment group, time, and OI type) showed that the trend in treatment response in LS aBMD over the course of the study was significantly different in patients with type I versus type III/IV OI; aBMD increased in type I patients $(P=0.0001)$, but did not in type III/IV patients $(P=0.98)$. Moreover, interaction analyses demonstrated that type I patients had significant treatment effects at 12 and 18 months $(P=0.04$ and
$P=0.002$, respectively), while those with type III/IV had no response at any time point. The interaction analysis also showed that type I patients had a more robust response to therapy than type III/IV patients at 18 months $(P=0.02)$.

In patients with type I OI, vertebral vBMD increased with teriparatide treatment and declined in the placebo group $(24.5 \% \pm 7.3 \%$ vs. $-5.5 \% \pm 7.3 \%$ change; $P=0.008$; Supplemental Figure $2 \mathrm{~A})$. Estimated vertebral strength increased in the teriparatide versus the placebo group $(18.7 \% \pm 4.6 \%$ vs. $-3.1 \% \pm 4.3 \%$ change; $P=0.003)$, whereas $\Phi$ declined $(-12.9 \% \pm 3.6 \%$ vs. $3.1 \% \pm 3.4 \%$ change; $P=0.004)$. Changes in these measures also occurred in type III/IV OI patients in response to teriparatide (Supplemental Figure 2B), but were not significantly different than placebo. The interaction terms between OI type and treatment response were not significant (vBMD, $P=0.24$; strength, $P=0.19 ; \Phi, P=0.23$ ), although there were relatively few participants with these quantitative computed tomography-based (QCT-based) measures.

Bone remodeling markers. In the placebo-treated group, levels of all bone remodeling markers remained essentially stable. With teriparatide therapy, P1NP levels increased rapidly, with a maximum at month $12(134.6 \% \pm 14.2 \%$ change $)$ and declined somewhat thereafter, but were always significantly higher than 


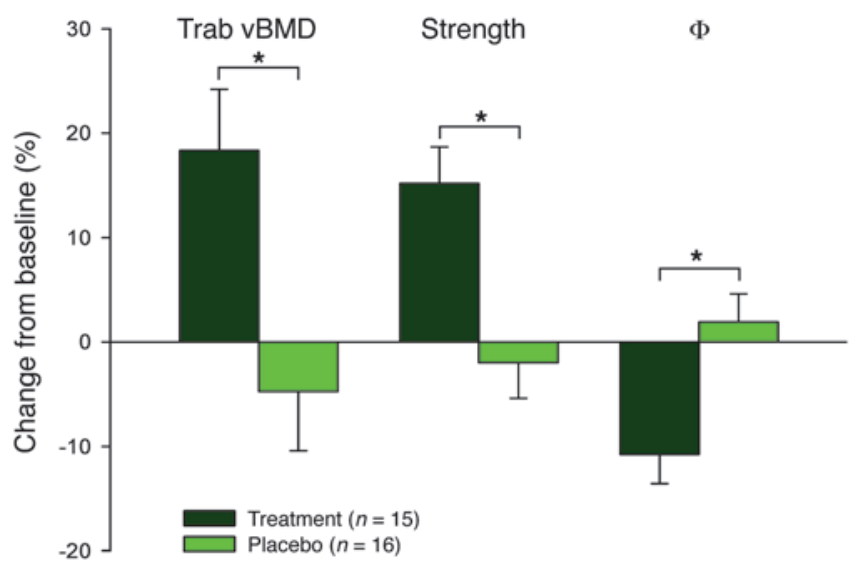

Figure 3

Spine QCT measures and FEA. Percent change from baseline in spinal trabecular vBMD $\left(\mathrm{mg} / \mathrm{cm}^{3}\right)$, vertebral strength $(\mathrm{N})$, and $\Phi$ (vertebral load/vertebral strength ratio) in teriparatide-and placebo-treated patients (mITT population) at 18 months. Error bars denote SEM. ${ }^{*} P<0.05$ between treatment groups.

placebo (Figure 4A). There was a slower increase in urine $\mathrm{N}$-terminal telopeptide of type 1 collagen (NTx); maximum levels occurred at month $6(63.6 \% \pm 10.0 \%$ change $)$ and were similar thereafter (Figure 4B).

Post-hoc analyses of subgroup differences showed that in patients with type I OI, biochemical marker levels were stable in the placebo-treated group (Supplemental Figure 3, A and B), while changes with teriparatide were similar to those in the pooled analyses. The extent to which LS and TH aBMD increased at 18 months positively correlated with the increase in serum P1NP at $3(r=0.54, P=0.0001)$ and $12(r=0.50, P=0.001)$ months. In patients with type III/IV OI, serum P1NP also increased significantly with teriparatide therapy (Supplemental Figure 3C), but the increases were less marked than those observed in patients with type I OI ( $82 \%$ vs. $163 \%$ change at 12 months; $P=0.005)$ and were not correlated with BMD change. An interaction analysis showed that the increases in P1NP in response to teriparatide were significant in the type I group $(P<0.001)$, but not in the type III/IV group $(P=0.87)$. Teriparatide-induced increases in urine NTx in patients with type III/IV OI were proportionately greater at 6 months than the increases in type I OI (79\% vs. 55\% change), but not significantly so $(P=0.25)$, and NTx levels appeared to decline more in the type III/IV group at later visits (Supplemental Figure 3, $\mathrm{B}$ and D). Interaction analyses supported that trend: type III/IV patients showed a significant treatment response at early followup visits (1, 3, and 6 months), but levels were not significantly different from baseline at 18 months. The NTx increase in type I subjects was significant at 3 months and remained so throughout the rest of the trial. 3-way interaction analyses were not significant for P1NP $(P=0.5)$ or NTx $(P=0.26)$.

Mineral metabolism. PTH concentrations declined by approximately $30 \%$ at 1 and 3 months in the teriparatide-treated group, but thereafter returned to levels similar to those of the placebotreated group (Supplemental Figure 4A). Serum calcium levels were stable, and no episodes of hypercalcemia were observed. Mean 24-hour urine calcium excretion remained stable with placebo, but increased with teriparatide (Supplemental Figure 4B); 13 teriparatide-treated patients were noted to have at least 1 episode of 24-hour urine calcium exceeding $300 \mathrm{mg} / 24 \mathrm{~h}$.

Fractures. 11 patients in the teriparatide treatment group (29\%) and 14 in the placebo group (36\%) reported fractures during the study (odds ratio [OR], 0.73; 95\% CI, 0.28-1.90). 1 patient had missing data on fracture at all visits. Of the 50 patients with type I OI, 8 subjects in the teriparatide treatment group (33.3\%) and 9 in the placebo group (34.6\%) reported fractures (OR, 0.94; $0.29-3.05)$. Of 26 patients with type III/IV OI, 3 subjects in the teriparatide treatment group $(21.4 \%)$ and 5 in the placebo group (41.7\%) reported fractures (OR, 0.38; 95\% CI, 0.07-2.13). Change in aBMD was not associated with the likelihood of fracture.

Safety. Teriparatide was well tolerated. No differences were observed between the treatment and placebo groups in the occurrence of serious adverse events.

\section{Discussion}

This was the largest placebo-controlled treatment trial in adults with OI, and the first trial of anabolic therapy in this disease. In patients with less severe OI (type I), teriparatide resulted in increased markers of bone formation, accompanied by increases in proximal femur and LS aBMD. Moreover, vertebral vBMD was considerably increased, and there was a comparable increase in estimated vertebral strength. These results indicated that despite an underlying genetic defect in OI that impairs bone matrix synthesis and results in osteoblastic dysfunction and remodeling abnormalities, anabolic therapy is capable of increasing bone formation and bone mass. However, exploratory analyses suggested that effects were less positive in patients with severe OI (type III/IV), potentially indicating that anabolic therapy may be less effective in this subset of patients.

Participants in this trial had previously experienced multiple fractures, and most had never received therapy. Unfortunately, there are few treatment trials in adults with OI. Although bisphosphonates appear to increase BMD in adults with OI $(6,14)$, they reduce bone remodeling and bone formation, actions that may be problematic in the presence of underlying defects in bone formation and osteoblastic function. In contrast, in our study of teriparatide therapy, there was an anabolic pattern of change in remodeling markers that mimicked those observed in previous studies of teriparatide therapy in osteoporosis (17). Moreover, the teriparatide-induced improvements in aBMD and vertebral vBMD in the type I OI patients were similar to those seen in osteoporosis $(18,19)$ and similar to the responses recently reported in a small clinical experiment with teriparatide therapy in type I OI (20). This skeletal response to teriparatide therapy has been associated with a marked reduction in fracture risk in patients with osteoporosis. The increases in bone density and strength in our patients are particularly noteworthy in light of the decline in TH aBMD and vertebral strength in the placebo-treated group. The anabolic response observed in type I OI sets the stage for more definitive studies with sufficient power to assess the effect of teriparatide therapy on fracture risk. In addition, since bisphosphonate therapy can increase BMD in adults with OI, our results raise the possibility that combination therapy with teriparatide and bisphosphonates may be useful. Combined therapy has been shown by some to be useful in osteoporosis (21).

Changes in BMD were most apparent at more trabecular sites (aBMD at the LS and TH, vBMD of the spine), whereas at the FN and radial measurement sites, there were no obvious changes with therapy. This pattern has been previously reported in teriparatide-treated patients with osteoporosis $(16,18)$. Despite the lack 

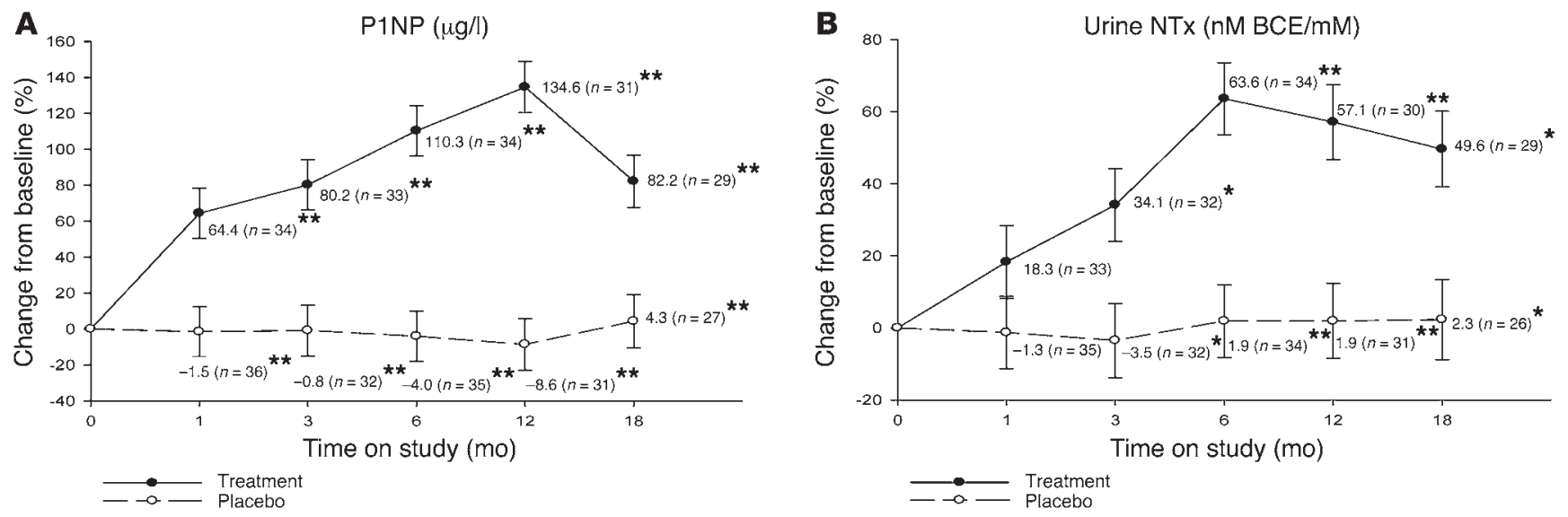

Figure 4

Bone remodeling markers. (A and B) Percent change from baseline in serum P1NP ( $\mu \mathrm{g} / \mathrm{l} ; \mathbf{A})$ and urinary NTx (nM BCE/mM; B) in teriparatide and placebo-treated patients (mITT population) at baseline and at 6, 12, and 18 months. Error bars denote SEM. Values shown are estimated least-squares mean of percent change. The number of patients with nonmissing percent change data at each time point is shown in parentheses. ${ }^{\star} P<0.05,{ }^{*} P<0.001$ between treatment groups.

of increase in BMD at the more cortical sites, it has been speculated that other effects of teriparatide may yield an improvement in bone strength in those areas, resulting in reduced fracture risk (22). Whether similar effects accrued in our patients is unknown. Although the placebo group may have experienced small changes in FN or radial aBMD in the later part of the study (Figure 2, C and D), those changes were small and nonsignificant.

Our data suggest the possibility of a differential treatment response in patients with quantitative defects of collagen (type I OI) versus those with qualitative collagen defects in OI (type III/IV). A priori, stimulating remodeling activity may not be efficacious in the context of the abnormal matrix production and cellular dysfunction that may be caused by qualitative collagen defects. For example, a reported increase in unfolded protein response in a murine model of OI may worsen in situations where osteoblastic function is stimulated $(23,24)$. In fact, in the patients with more severe OI, there was a blunted increase in serum P1NP and an exaggerated increase in urine NTx, and changes in BMD were not different than those observed with placebo, which suggests that teriparatide may have a less favorable effect in more severe disease. These findings raise the concern that more severe defects in matrix and osteoblast function may preclude the usefulness of anabolic therapies that do not address the underlying molecular defect in collagen production. On the other hand, in a mouse model of OI thought to be phenotypically similar to type IV disease in humans $\left(\mathrm{Brtl}^{+/-}\right.$mice), Sinder et al. recently reported that anti-sclerostin antibody treatment induced a positive response, including reduced long bone fragility and preserved tissue mineralization properties (25). The subgroup differences we observed must be considered preliminary; the number of participants studied was small, and the subgroup analyses were not part of the predetermined statistical approach. Nevertheless, these results suggest that teriparatide therapy might be of more clinical utility in patients with milder forms of OI. If so, characterizing quantitative versus qualitative defects of collagen synthesis may help in patient selection for therapy. Additional clinical trials designed to test subgroup differences in the response to therapy are needed.

This study has both strengths and limitations. We studied sufficient numbers of patients with type I OI to confidently detect a meaningful change in BMD. In addition, we used FEA to assess vertebral biomechanical strength. On the other hand, the number of patients we studied with type III/IV was limited, and we can be less certain of the findings in that group. Genotypic characterization of the patients was not available; hence, responses based on qualitative versus quantitative defects in collagen production could not be assessed. The trial was not powered to adequately assess the effect of teriparatide on fracture risk; although fewer fractures were reported in those treated with teriparatide than placebo, the difference was not significant. Nevertheless, our results indicate that additional studies are warranted to evaluate anabolic therapies in reducing fracture risk in adults with OI.

In conclusion, teriparatide therapy significantly increased bone density and estimated bone strength in adults with a mild form of OI (type I). These data should prompt larger trials to evaluate whether anabolic treatment results in a reduction in the burden of fractures in those patients. On the other hand, our results suggest that anabolic therapies may be less effective in patients with more severe forms of OI, and future studies should specifically assess the usefulness of therapies in light of disease severity.

\section{Methods}

Participants. At 3 academic medical centers, patients were recruited from registries and by publicizing the trial in partnership with advocacy groups. Patients $\geq 18$ years of age with a well-established clinical diagnosis of OI, characteristic physical findings, and fused epiphyses were eligible for enrollment.

Exclusion criteria included the following: current therapy with osteoporosis medications or antiresorptive therapy in the previous year $(26 \mathrm{had}$ received bisphosphonates in the past, all for $<2$ years and 14 for $<1$ year); creatinine clearance $<30 \mathrm{ml} / \mathrm{min}$; serum alkaline phosphatase $>1.5 \times$ upper limit of normal (ULN), or aspartate aminotransferase/alanine aminotransferase > $3 \times$ ULN; hypercalcemia or hypocalcemia; abnormal thyroid function. Other osteoporosis medications or systemic corticosteroids were prohibited during the trial. Subjects who had 25-hydroxy $(\mathrm{OH})$ vitamin D levels $<15 \mathrm{ng} / \mathrm{ml}(37.4 \mathrm{nmol} / \mathrm{l})$ were eligible only if supplementation yielded subsequent measures that exceeded that threshold.

Study design. This was an 18-month, randomized, double-blinded, placebocontrolled, parallel-group study conducted from November 2004 to Novem- 
ber 2010. Participants were randomized at a 1:1 ratio, without the use of additional stratification variables, to receive subcutaneous teriparatide $(20 \mu \mathrm{g} / \mathrm{d}$; Eli Lilly and Co.) or placebo. After baseline studies, return visits occurred at $1,3,6,12$, and 18 months. During the trial, all participants received daily supplements of calcium (500 mg, twice daily) and vitamin D (800 IU).

A priori power estimates suggested that randomization of 90 participants, assuming a $15 \%$ subsequent dropout rate, were required to confidently demonstrate ( $80 \%$ power, 2 -tailed $\alpha=0.05$ ) the efficacy of therapy ( $5 \%$ difference between treatment and placebo groups) on the primary outcome variable, LS aBMD.

A formal interim analysis was performed when 48 participants had completed the protocol. It revealed that a significant difference would be achieved in the primary endpoint with fewer numbers of participants than anticipated, but that it was highly unlikely that a difference in fracture rate would result, even if the trial was fully enrolled. At that point, a decision was made to discontinue new recruitment and to continue follow-up of enrolled participants. The analyses presented are the results obtained with complete follow-up of those patients.

Study measurements. aBMD at the LS, TH, FN, and radius were assessed by dual-energy X-ray absorptiometry (DXA). All study sites used the same DXA machine (Hologic QDR4500). The software versions used were as follows: site 1 , versions 12.3 and 12.6 ; site 2 , versions $12.3,12.4$, and 12.6 ; site 3 , versions 11.2 and 12.1 . These versions are not different in essential elements of the BMD assessments, and differences in versions were necessary primarily to allow BMD measures at very low density. The software version used was consistent in BMD assessments in an individual participant, with the exception that several participants required the use of a software version for low density. Analyses were performed using the manufacturer's protocols, including those appropriate for altered skeletal anatomy. No obviously fractured vertebrae were included in the analyzed scan fields. All scans were reviewed centrally in a blinded fashion, and reanalyzed if necessary to ensure consistency. 1 participant had spinal deformity, and 13 had deformities or surgical hardware in the proximal femurs, precluding adequate DXA analyses. Radial aBMD was unavailable in 28 participants due to previous surgery or fracture or poor scan quality.

Spinal QCT scans were performed on 31 participants at baseline and 18 months. QCT scans were not available for 26 participants who had DXA results, for the following reasons: 10 did not undergo QCT scans; 8 had 1 QCT scan, but not at both baseline and final visits; 8 had scans obtained, but technical reasons prevented their analysis (surgical hardware, skeletal deformity). vBMD was assessed in the central portion of the vertebral body (19). Patient-specific L1 vertebral strength for a simulated compression overload was estimated using nonlinear 3D FEA (VirtuOst, ON Diagnostics) $(19,26)$. These estimates have previously been shown to be in excellent agreement with measured strength from biomechanical cadaver experiments $(27)$ and to predict prevalent $(28,29)$ and incident $(27)$ vertebral fractures. $\Phi$ was calculated as the ratio of estimated force placed on a vertebra during loaded flexion to the estimated vertebral strength by FEA (28).

We measured fasting morning urine NTx (Ostex International), serum P1NP (Immuno Diagnostic Systems), and PTH(1-34) (Immulite, Siemens Healthcare Diagnostics). Serum calcium, phosphorus, creatinine, and 25 -hydroxy $(\mathrm{OH})$ vitamin $\mathrm{D}$, as well as 24 -hour urine calcium and creatinine, were measured using conventional methods. Clinical fractures (vertebral and nonvertebral) were reported by patients at each visit, but were not verified by X-ray report or medical records. At each visit, adverse events were monitored, recorded, and coded using the Medical Dictionary for Regulatory Activities system.

Statistics. The primary endpoint was percent change in LS aBMD. Secondary endpoints included percent change in TH aBMD, FN aBMD, radial $\mathrm{aBMD}$, vertebral $\mathrm{vBMD}$, vertebral strength, and bone remodeling markers; proportion of participants reporting incident fractures; and teriparatide safety. The power calculations for this study assumed a 0.1 SD of BMD and dropout rate of $15 \%$. The study was designed to have $80 \%$ power to detect a between-group difference of $5 \%$ change in LS aBMD at the 2-sided $5 \%$ significance level. Analysis of efficacy was performed on the mITT population (patients who had baseline and at least 1 post-baseline BMD assessments). For each patient, the effect of treatment on percent change in aBMD, vBMD, and bone turnover markers was estimated with separate generalized linear mixed models. In each model, the dependent variable was percent change from baseline. The model included group, visit time, and group-time interaction entered as fixed effects; the intercept was entered as a random effect; and variance components structure was used for the within-subject correlation. Since vBMD and vertebral strength were only assessed at baseline and 18 months, percent change from baseline to month 18 was compared between the 2 treatment groups using generalized linear models (30). Estimated least squares mean and SEM in percent change from baseline was plotted over time. A 3-way interaction was used to examine whether the trend of LS aBMD change over time in the treatment and placebo groups differed by OI type. If significant interaction was present, we investigated the interaction further by computing the simple main effect via the SLICE option in PROC MIXED (31). This statement creates partitions of interaction effect and assesses the significance of a given factor (e.g., visit time) at each combination of levels of the other 2 factors (e.g., treatment group and OI type). Therefore, comparisons were made between the treatment and placebo groups for different OI types at each follow-up visit in order to localize which combinations of visit and OI type showed treatment differences. The association of treatment group and fracture incidence was estimated using a logistic regression model and expressed as OR with $95 \%$ CI. For all tests, 2 -sided $P$ values less than 0.05 were considered significant. All analyses were performed using SAS version 9.3 (SAS Institute).

Study approval. The protocol was approved by the local institutional review boards. All participants provided written informed consent.

\section{Acknowledgments}

The study was funded by the Osteogenesis Imperfecta Foundation and an investigator-initiated grant from Eli Lilly and Co. It was supported by the National Center for Advancing Translational Science (NCATS) at the NIH (grant no. UL1RR024140) and by the Baylor College of Medicine General Clinical Research Center (grant no. RR00188). S.C.S. Nagamani was supported through fellowship grants by the Osteogenesis Imperfecta Linked Clinical Research Centers, National Urea Cycle Disorders Foundation, and a Clinical Scientist Development Award from the Doris Duke Charitable Foundation. The authors have high regard for personal contributions of all those with OI who participated in the trial. They also acknowledge the invaluable assistance of Robert Steiner for initial discussions about the protocol design, the staffs of the clinical research centers that supported the patient visits (especially the core lab staff at the Oregon Health and Science University Clinical and Translational Research Center), Catherine Pedersen for complex grants management, and Denise Duncan for expert manuscript preparation.

Received for publication May 30, 2013, and accepted in revised form November 5, 2013.

Address correspondence to: Eric S. Orwoll, Oregon Health and Science University, 3181 SW Sam Jackson Park Road, Portland, Oregon 97239, USA. Phone: 503.494.1385; Fax: 503.494.4816; E-mail: orwoll@ohsu.edu. 
1. Cundy T. Recent advances in osteogenesis imperfecta. Calcif Tissue Int. 2012;90(6):439-449.

2. Marini JC, Blissett AR. New genes in bone development: what's new in osteogenesis imperfecta. J Clin Endocrinol Metab. 2013;98(8):3095-3103.

3. Folkestad L, et al. Bone geometry, density, and microarchitecture in the distal radius and tibia in adults with osteogenesis imperfecta type I assessed by high-resolution PQCT. J Bone Miner Res. 2012 27(6):1405-1412

4. Gatti D, et al. The volumetric bone density and cortical thickness in adult patients affected by osteogenesis imperfecta. J Clin Densitom. 2003;6(2):173-177.

5. Bradbury LA, et al. Risedronate in adults with osteogenesis imperfecta type I: increased bone mineral density and decreased bone turnover, but high fracture rate persists. Osteoporos Int. 2012; 23(1):285-294.

6. Shapiro JR, Thompson CB, Wu Y, Nunes M, Gillen $C$. Bone mineral density and fracture rate in response to intravenous and oral bisphosphonates in adult osteogenesis imperfecta. Calcif Tissue Int. 2010;87(2):120-129.

7. Dimeglio LA, Ford L, McClintock C, Peacock M. A comparison of oral and intravenous bisphosphonate therapy for children with osteogenesis imperfecta. J Pediatr Endocrinol Metab. 2005;18(1):43-53.

8. Gatti D, et al. Intravenous neridronate in children with osteogenesis imperfecta: a randomized controlled study. J Bone Miner Res. 2005;20(5):758-763.

9. Glorieux FH, et al. Alendronate in the treatment of pediatric osteogenesis imperfecta [abstract]. J Bone Miner Res. 2004;19:S12.

10. Letocha $\mathrm{AD}$, et al. Controlled trial of pamidronate in children with types III and IV osteogenesis imperfecta confirms vertebral gains but not short-term functional improvement. J Bone Miner Res. 2005; 20(6):977-986

11. Sakkers R, et al. Skeletal effects and functional outcome with olpadronate in children with osteogen- esis imperfecta: a 2-year randomised placebo-controlled study. Lancet. 2004;363(9419):1427-1431.

12. Seikaly MG, et al. Impact of alendronate on quality of life in children with osteogenesis imperfecta. J Pediatr Orthop. 2005;25(6):786-791.

13. Bishop $N$, et al. Risedronate in children with osteogenesis imperfecta: a randomised, doubleblind, placebo-controlled trial. Lancet. 2013; 382(9902):1424-1432.

14. Chevrel G, et al. Effects of oral alendronate on BMD in adult patients with osteogenesis imperfecta: a 3-year randomized placebo-controlled trial. J Bone Miner Res. 2006;21(2):300-306

15. Adami $\mathrm{S}$, et al. Intravenous neridronate in adults with osteogenesis imperfecta. J Bone Miner Res. 2003; 18(1):126-130

16. Jiang Y, Zhao JJ, Mitlak BH, Wang O, Genant HK, Eriksen EF. Recombinant human parathyroid hormone (1-34) [teriparatide] improves both cortical and cancellous bone structure. J Bone Miner Res. 2003;18(11):1932-1941.

17. Uihlein AV, Leder BZ. Anabolic therapies for osteoporosis. Endocrinol Metab Clin North Am. 2012; 41(3):507-525.

18. Neer RM, et al. Effect of parathyroid hormone (1-34) on fractures and bone mineral density in postmenopausal women with osteoporosis. NEngl JMed. 2001;344(19):1434-1441.

19. Keaveny TM, Donley DW, Hoffmann PF, Mitlak $\mathrm{BH}$, Glass EV, San Martin JA. Effects of teriparatide and alendronate on vertebral strength as assessed by finite element modeling of QCT scans in women with osteoporosis. J Bone Miner Res. 2007;22(1):149-157.

20. Gatti D, et al. Teriparatide treatment in adult patients with osteogenesis imperfecta type I. Calcif Tissue Int. 2013;93(5):448-452.

21. Cosman F, et al. Hip and spine strength effects of adding versus switching to teriparatide in postmenopausal women with osteoporosis treated with prior alendronate or raloxifene. J Bone Miner
Res. 2013;28(6):1328-1336.

22. Zanchetta JR, et al. Effects of teriparatide [recombinant human parathyroid hormone (1-34)] on cortical bone in postmenopausal women with osteoporosis. J Bone Miner Res. 2003;18(3):539-543.

23. Bianchi L, et al. Differential response to intracellular stress in the skin from osteogenesis imperfecta Brtl mice with lethal and non lethal phenotype: a proteomic approach. J Proteomics. 2012; 75(15):4717-4733.

24. Forlino A, et al. Differential expression of both extracellular and intracellular proteins is involved in the lethal or nonlethal phenotypic variation of BrtlIV, a murine model for osteogenesis imperfecta. Proteomics. 2007;7(11):1877-1891.

25. Sinder BP, Eddy MM, Ominsky MS, Caird MS, Marini JC, Kozloff KM. Sclerostin antibody improves skeletal parameters in a $\mathrm{Brtl} /+$ mouse model of osteogenesis imperfecta. J Bone Miner Res. 2013; 28(1):73-80.

26. Crawford RP, Cann CE, Keaveny TM. Finite element models predict in vitro vertebral body compressive strength better than quantitative computed tomography. Bone. 2003;33(4):744-750.

27. Wang $X$, et al. Prediction of new clinical vertebral fractures in elderly men using finite element analysis of CT scans. J Bone Miner Res. 2012;27(4):808-816.

28. Melton LJ, et al. Structural determinants of vertebral fracture risk. J Bone Miner Res. 2007; 22(12):1885-1892.

29. Melton LJ, et al. Relation of vertebral deformities to bone density, structure, and strength. J Bone Miner Res. 2010;25(9):1922-1930.

30. SAS Institute. SAS/STAT 9.3 User's Guide. SAS Web site. http://support.sas.com/documentation/cdl/en/statug/63962/HTML/default/viewer. htm\#titlepage.htm. Accessed December 9, 2013

31. Littell RC, Milliken GA, Stroup WW, Wolfinger RD. SAS System for Mixed Models. Cary, North Carolina, USA: SAS Institute Inc.; 1996. 